(C) 1981. The Genetical Society of Great Britain

\title{
THE EVOLUTION OF APOMIXIS
}

\author{
D. R. MARSHALL and A. H. D. BROWN \\ CSIRO, Division of Plant Industry, P.O. Box 1600, Canberra City, Australia
}

Received 23,x.80

\section{SUMMARY}

\begin{abstract}
The selective forces responsible for the evolution of gametophytic apomixis in outbreeding plant populations are analysed in terms of a simple single gene model. In the absence of selection, apomixis inevitably becomes fixed in a population. This conclusion holds regardless of the dominance relations of the alleles specifying apomictic versus sexual seed formation. Substantial heterotic viability selection is required to prevent fixation of recessive and codominant apomictic alleles and maintain a stable polymorphism at the mating system locus. These findings suggest that gametophytic apomixis should be a common mode of reproduction in plant species. Possible factors accounting for the relative paucity of apomictic plants are discussed. It is concluded that one of the major factors hindering the spread of apomixis is its usually complex inheritance and the need to accumulate, in the one individual, two or more mutations affecting meiosis and the reproductive system.
\end{abstract}

\section{INTRODUCTION}

AgAMOSPERMY, asexual reproduction through seed formation, was first recorded by Smith in 1841 in Alchornea ilicifolia from Australia (Gustafsson, 1946-47). Since then, some 250 species belonging to 22 families have been found to reproduce wholly or partly by agamospermy via a variety of embryological pathways (reviews in Nygren, 1954, 1967; Battagalia, 1963; Grant, 1971).

The wide taxonomic distribution of agamospermy and the multiplicity of mechanisms underlying asexual seed formation suggest that this mode of reproduction has arisen independently on several different occasions in higher plants. Research over the last century has provided some insights into the evolution of this form of apomixis. In particular, it has shown that agamospermy, like other features of the breeding system of plants, is under genetic control and is usually governed by two or more loci (Asker, 1979). It has also shown that most agamospermous species are facultative apomicts and that environmental factors can effect the level of sexuality. Further, past studies indicate that agamospermous species are usually perennial and derived from strongly outcrossing ancestors. They also show that agamospermy is often, but by no means invariably, associated with interspecific hybridization and polyploidy (Gustafsson, 1946, 1947a, $b$; Stebbins, 1950; Nygren, 1954, 1967; Knox, 1967; Knox and Heslop-Harrison, 1963; Grant, 1971).

However, the evolution of agamospermy, or indeed, the whole question of the evolution of sexual versus asexual reproduction, remains a highly controversial area of research (Maynard Smith, 1978). This controversy is reflected in the fact that three contrasting viewpoints have appeared in the literature regarding the adaptive significance of apomixis and its establishment in plant populations. Each of these is considered briefly below. 


\section{Current Theories}

(i) The "escape from sterility" hypothesis

One of the earliest theories, first proposed by Darlington (1939) and promoted by Stebbins $(1941,1950)$, is that agamospermy offers no real selective advantage over normal sexual reproduction. Indeed, it was argued that it restricts recombination and hence, the evolutionary potential in species in which it becomes established. From this viewpoint the only, or at least principal, adaptive advantage of apomixis is that it restores the fertility of individuals that would otherwise be sexually sterile as a consequence of polyploidy and hybridization. This theory achieved wide currency because it was compatible with the then prevalent view that sexual reproduction greatly accelerated the rate of adaptive evolution and was maintained in species by interpopulational or group selection (Fisher, 1930; Muller, 1932; Crow and Kimura, 1965), and also because it provided a ready explanation for the close association between apomixis, polyploidy and interspecific hybridization. It has lost favour in recent years, with the development of a variety of conflicting theories. However, it still has its advocates. For example, de Wet and Stalker (1974) wrote "Apomixis is not necessary. Neither agamospermy nor its common companion polyploidy confer selective advantages on populations that are not equally well provided for in the diploid sexual breeding system. ... The principal adaptive advantage of apomixis is that it can restore fertility in sexually sterile individuals".

\section{(ii) The "Henry Ford" or "Model T" hypothesis}

Clausen (1954) was among the first to recognize formally that apomixis, particularly facultative apomixis, does not necessarily lead to a loss of variation and evolutionary potential. Indeed, using an analogy between the adaptive process in facultative agamosperms and the mass production of automobiles, he argued that a combination of sexuality, which allows for the progessive production of new genotypes, and apomixis, which permits the unlimited and faithful reproduction of the best of the genotypes generated, would enhance rather than diminish a species' capacity for adaptive change.

The hypothesis that partial apomixis offered more than a short-term escape from sterility was also widely accepted and has become the dominant view (Heslop-Harrison, 1961; Grant, 1971). It is supported by findings that the level of apomixis in a number of facultative species varies, presumably adaptively, with environmental conditions (Knox and HeslopHarrison, 1963; Knox, 1967; Saran and de Wet, 1970). However, it suffers from the disadvantages that it is not quantitative, although this deficiency is being rectified (Asher, 1970; Marshall and Weir, 1979) and it ignores the question of the "cost of meiosis" (Williams, 1975) in relation to the establishment of apomixis in plant species.

\section{(iii) The automatic advantage hypothesis}

This hypothesis developed from recent quantitative studies designed to resolve the highly controversial question of the evolution of sex (Crow and 
Kimura, 1965, 1969; Maynard Smith, 1968, 1971a, 1976, 1978; Williams and Mitton, 1973; Williams, 1975). These studies show that asexual reproduction has an automatic two-fold advantage over sexual reproduction. This two-fold advantage can accrue because of either within population (individual) selection or between population (group) selection.

The two-fold advantage of asexual reproduction at the individual level arises because all the offspring of a parthenogenetic or apomictic parent carry the full genetic complement of their mother. Sexual outcrossed offspring, on the other hand, carry only half the genetic complement of their maternal parent. If both sexual and asexual parents produce equal numbers of offspring, then the asexual maternal parent will have double the genetic representation of the sexual in the offspring. The same situation applies to the next generation. Both genes of the asexual mother will be present in all her grandchildren, while only a quarter of the genes of the sexual mother will be present in her grandchildren. Each gene of a sexual outcrossing individual suffers a 50 per cent hazard per generation compared to the asexual alternative (Williams, 1975).

The twofold advantage of parthenogenesis at the interpopulational or group level arises because no resources need be wasted on males, or male gametes. We would expect, therefore, that asexual species could produce twice as many female progeny with the same level of parental investment as dioecious sexual species producing equal numbers of male and female progeny (Maynard Smith, 1971a, 1977) and should, as a group, be at an evolutionary advantage.

The disadvantage of sexual reproduction at both the individual and the group level has been referred to as the "cost of meiosis", although they have entirely different origins.

\section{Basic Genetic Models}

A number of phenotypic (Williams, 1975; Lloyd, 1977) as well as genetic (Maynard Smith, 1971b; Charlesworth, 1980) models have been used to study various aspects of the evolution of asexual reproduction in animal and plant populations. Here we consider a simple genetic model for the evolution of apomixis analogous to the "pollen elimination" model of the evolution of autogamy first developed by Fisher (1941) and analysed in more detail by Moran (1962) and Jain and Workman (1967). We chose this model for three reasons. First, it is applicable to a wide range of species, particularly hermaphroditic outbreeders, which have been the main progenitors of apomictic species in plants (Gustafsson, 1946; Stebbins, 1950). Second, elegant mathematical techniques for the analysis of this model have been developed previously by Workman and Jain (1966) and Jain and Workman (1967). Third, the results of the present analysis should prove more informative when considered in comparison to known results for the inbreeding model rather than in isolation.

We consider an infinite diploid population in which the mating system is governed by a single diallelic locus (with alleles $A_{1}, A_{2}$ ). Allele $A_{1}$ specifies sexual reproduction with random mating and its counterpart $A_{2}$ specifies agamospermy. It is assumed throughout that all genotypes produce pollen equally well and that this pollen is distributed at random over the 
population. It is also assumed, in the first instance, that all genotypes are equally fit.

Three variations of this model differing in the dominance relations of $A_{1}$ and $A_{2}$ are considered below.

\section{(a) Apomixis recessive}

Consider the case where genotypes $A_{1} A_{1}$ and $A_{1} A_{2}$ mate at random while $A_{2} A_{2}$ is obligately apomictic. If the relative genotypic frequencies at the mating system locus are denoted $P\left(A_{1} A_{1}\right), R\left(A_{1} A_{2}\right)$ and $Q\left(A_{2} A_{2}\right)$, the recurrence equations relating genotypic frequencies in successive generations for this model are:

$$
\begin{aligned}
& A_{1} A_{1}: P^{\prime}=(P+R / 2)^{2} \\
& A_{1} A_{2}: R^{\prime}=R / 2+P(Q+R / 2) \\
& A_{2} A_{2}: Q^{\prime}=Q+(R / 2)(Q+R / 2) .
\end{aligned}
$$

From the above equations the relative gene frequencies, $p\left(A_{1}\right)$ and $q\left(A_{2}\right)$, in successive generations are given by:

$$
\begin{aligned}
& A_{1}: p^{\prime}=p-p Q / 2 \\
& A_{2}: q^{\prime}=q+p Q / 2 .
\end{aligned}
$$

The change in gene frequency per generation is

$$
\Delta p=-p Q / 2
$$

so that, at equilibrium

$$
p=0 \text { or } Q=0,
$$

and it is clear that the allele specifying sexual reproduction will be lost unless it is initially fixed in the population.

As noted by Charlesworth (1980) a recessive mutation to apomixis has a vanishingly small initial advantage because of the rarity of the recessive homozygote $\left(\mathrm{A}_{2} \mathrm{~A}_{2}\right)$ so that such genes are unlikely to become established in a large population of a random mating species. If they do become established in a measurable frequency, however, due to drift in small populations or migration from other populations then they will eventually become fixed in the population because of their automatic advantage.

\section{(b) Apomixis and sexuality codominant}

In this case, $A_{1} A_{1}$ is entirely sexual, $A_{2} A_{2}$ is obligately apomictic and $A_{1} A_{2}$ produces half its progeny by outcrossing and half by apomixis. The recurrence equations relating genotypic proportions in successive generations under this system are:

$$
\begin{aligned}
& A_{1} A_{1}: P^{\prime}=(P+R / 4)(P+R / 2) \\
& A_{1} A_{2}: R^{\prime}=3 R / 4+P(Q+R / 2) \\
& A_{2} A_{2}: Q^{\prime}=Q+(R / 4)(Q+R / 2)
\end{aligned}
$$


the gene frequencies in successive generations are given by:

$$
\begin{aligned}
& A_{1}: p^{\prime}=p-p q / 2+R / 8 \\
& A_{2}: q^{\prime}=q+p q / 2-R / 8 .
\end{aligned}
$$

At equilibrium,

$$
\Delta p=-p q / 2+R / 8=0
$$

which yields an unstable equilibrium when $R=4 p q$ and a stable equilibrium when,

$$
p=0 \text { or } p=1 \text {. }
$$

That is, the gene specifying sexuality is either initially fixed in the population or it is eventually lost in competition with the allele specifying agamospermy.

In this case, an apomictic mutant will possess a considerable initial advantage over its sexual alternative. When $A_{2}$ is rare $(p \simeq 1, R \simeq 2 q)$ then (4) above becomes:

$$
q^{\prime}=\frac{5}{4} q
$$

so that the initial advantage of a codominant apomictic mutant is $\frac{5}{4}$ rather than 2 as found by Maynard Smith $(1971 b)$ for a dominant asexual mutant in a dioecious species.

\section{(c) Apomixis dominant}

Here, genotypes $A_{1} A_{2}$ and $A_{2} A_{2}$ are assumed to be obligately apomictic while $A_{1} A_{1}$ is fully sexual. In this circumstance, the genotypic recurrence relations are:

$$
\begin{aligned}
& A_{1} A_{1}: P^{\prime}=P(P+R / 2) \\
& A_{1} A_{2}: R^{\prime}=R+P(Q+R / 2) \\
& A_{2} A_{2}: Q^{\prime}=Q
\end{aligned}
$$

and these yield

$$
\begin{aligned}
& A_{1}: p^{\prime}=p-q P / 2 \\
& A_{2}: q^{\prime}=q+q P / 2
\end{aligned}
$$

at equilibrium we have for this model,

$$
\Delta p=-q P / 2=0
$$

and we have

$$
q=0 \text { or } P=0
$$

that is, there are no $A_{2}$ alleles specifying apomixis in the initial population, or,

$$
Q+R=1
$$

in which case all plants would be apomictic but the mating system locus would be polymorphic. The equilibrium gene frequencies will depend on the initial values of $P$ and $Q$. 
If $P=1$ initially, the most likely source of an apomict will be a mutation producing a heterozygote $A_{1} A_{2}$, and in this case the population will eventually consist entirely of heterozygotes $(R=1)$. Here, when $A_{2}$ is rare initially $(P \simeq 1)$, (7) above becomes,

$$
q^{\prime} \simeq 3 q / 2 \text {. }
$$

It is evident that the initial advantage of a dominant asexual mutant in hermaphroditic species is $\frac{3}{2}$ rather than 2 as in dioecious species (Maynard Smith, 1971b). A similar conclusion was reached by Charlesworth (1980) who also demonstrated that the initial advantage of a dominant mutant varied with the level of inbreeding in the population.

\section{The EfFects of Selection}

It is clear from the preceding section that, once introduced, agamospermy will invariably become fixed in a population, regardless of the dominance relations of the alleles specifying apomictic versus sexual seed formation, unless it is opposed by some form of selection. Consequently, the above treatment will now be extended to include the effects of zygotic selection.

The above three models can be combined into one formulation by using an index variable, $k$, specifying the degree of dominance of the apomictic allele. When $k=0$, the allele for apomixis $\left(A_{2}\right)$ is recessive, when $k=\frac{1}{2}$ it is codominant, and when $k=1, A_{2}$ is dominant. To include selection, the relative viabilities of $A_{1} A_{1}: A_{1} A_{2}: A_{2} A_{2}$ are $W_{1}: 1: W_{3}$ respectively. Assuming genotypic frequencies are scored after all selection has occurred and just before mating, the recurrence relations become

$$
\begin{aligned}
& A_{1} A_{1}: P^{\prime}=W_{1}[P+R(1-k) / 2](P+R / 2) / \bar{W} \\
& A_{1} A_{2}: R^{\prime}=[R(1+k) / 2+P(Q+R / 2] / \bar{W} \\
& A_{2} A_{2}: Q^{\prime}=W_{3}[Q+R(Q+R / 2)(1-k) / 2] \bar{W} .
\end{aligned}
$$

The population mean fitness, $\bar{W}$, ensures that $P^{\prime}+Q^{\prime}+R^{\prime}=1$. Two trivial solutions for (8) are $\left(P=P^{\prime}=1\right.$, and $\left.Q=Q^{\prime}=R=R^{\prime}=0\right)$ and $\left(Q=Q^{\prime}=1\right.$, $P=P^{\prime}=R=R^{\prime}=0$ ). These are the boundary equilibria corresponding to fixation for sexuality or apomixis respectively. We now consider the case when $p q \neq 0$.

The most direct approach to the solution of these proportionalities is that of Workman and Jain (1966) and Jain and Workman (1967). This approach uses allele frequencies and Wright's fixation index $F$, where $F$ lies in the range $[-q / p,-p / q<F<1]$. If $1-F=R / 2 p q$ where $p=P+R / 2$ and $q=Q+R / 2$, then the genotypic distribution at any generation $[P, R, Q]$ can be written as $\left[p^{2}+p q F, 2 p q(1-F), q^{2}+p q F\right]$. The transformation (8) becomes

$$
\begin{aligned}
& P^{\prime}=p^{\prime 2}+p^{\prime} q^{\prime} F^{\prime}=W_{1} p^{2}[1-k q(1-F)] / \bar{W} \\
& R^{\prime}=2 p^{\prime} q^{\prime}\left(1-F^{\prime}\right)=p q[1+(p+k)(1-F)] / \bar{W} \\
& Q^{\prime}=q^{\prime 2}+p^{\prime} q^{\prime} F^{\prime}=W_{3} q[1-p(1-F)(p+q k)] / \bar{W}
\end{aligned}
$$


From (9) the recurrence relations for $p$ and $F$ follow as

$$
\begin{aligned}
p^{\prime} & =\left\{2 W_{1} p^{2}[1-k q(1-F)]+p q[1+(p+k)(1-F)]\right\} / 2 \bar{W} \\
1-F^{\prime} & =p q[1+(p+k)(1-F)] / 2 p^{\prime} q^{\prime} W
\end{aligned}
$$

where

$$
\begin{aligned}
\bar{W}= & W_{1} p^{2}[1-k q(1-F)]+p q[1+(p+k)(1-F)] \\
& +W_{3} q[1-p(1-F)(p+q k)] .
\end{aligned}
$$

At equilibrium $p^{\prime}=p$ and $F^{\prime}=F$, and equations (10) and (12) lead to

$$
1-F=\frac{1-2 W_{3}-2 p\left(1-W_{1}\right)}{2 W_{1} p q k+(p-q)(p+k)-2 W_{3} p(p+q k)}
$$

provided that the denominator is not zero, i.e.,

$$
2\left[1-W_{1} k-W_{3}(1-k)\right] p^{2}+\left[2 k\left(1+W_{1}-W_{3}\right)-1\right] p \neq k .
$$

Substituting (13) for $(1-F)$ in (12), and then (13) for $(1-F)$ and (12) for $W$ in (11), leads to a general fourth degree polynomial solution for $p$

$$
\begin{aligned}
2\{( & \left.W_{1}-W_{3}\right)\left(2-W_{1}-W_{3}\right)+2 W_{3}\left(1-W_{1}\right)^{2}-2 k\left[W_{3}\left(1-W_{1}\right)^{2}\right. \\
& \left.\left.+\left(W_{1}-W_{3}\right)\left(1-W_{3}\right)\right]+k^{2}\left(W_{1}-W_{3}\right)^{2}\right\} p^{4} \\
& +2\left\{2 W_{3}^{2}+2 W_{1} W_{3}-2 W_{1} W_{3}^{2}-W_{1}-W_{3}\right. \\
& +k\left[6 W_{1}-11 W_{1} W_{3}+2 W_{1} W_{3}^{2}+4 W_{1}^{2} W_{3}-W_{1}^{2}\right] \\
& \left.+k^{2}\left[W_{3}-W_{1}-3 W_{1}^{2}+5 W_{1} W_{3}-2 W_{3}^{2}\right]\right\} p^{3} \\
& +\left\{W_{3}\left(2 W_{1}-1\right)+k\left[8 W_{3}^{2}-4 W_{3}-5 W_{1}+10 W_{1} W_{3}-8 W_{1} W_{3}^{2}\right.\right. \\
& \left.\left.-4 W_{1}^{2} W_{3}\right]+k^{2}\left[5 W_{1}+4 W_{1}^{2}-3 W_{3}-4 W_{1} W_{3}\right]\right\} p^{2} \\
& +\left\{W_{3}\left(1-2 W_{3}\right)+4 k W_{1} W_{3}^{2}-k^{2}\left[2 W_{1}+W_{3}+2 W_{1} W_{3}-2 W_{3}^{2}\right]\right\} p \\
& +k W_{3}\left(1-2 W_{3}\right)+k^{2} W_{3}=0 .
\end{aligned}
$$

We consider only the specific cases $k=0, \frac{1}{2}$ and 1 .

(a) Apomixis recessive $(k=0)$

When $k=0$, the polynomial solution (15) for $p$ reduces to a cubic equation. Some particular solutions of (13) and (15) given $k=0$, for internal equilibria (i.e., $0<p<1 ;-q / p,-p / q<F<1$ ) are:

(i) If $W_{3}=0 \cdot 5$, then one internal equilibrium exists provided $W_{1}>0 \cdot 5$. This equilibrium is

$$
p=2 W_{1}-1 \text { and } F=0
$$

and is stable, whereas equilibria $p=0$ or $p=1$ are mutationally unstable. This result is of particular interest in that selection is such as to balance the advantage of apomixis. The consequence is a stable polymorphism for the apomictic gene, with the adult genotype frequencies in panmictic proportions. That $W_{3}=0.5, W_{1}>0.5$ is the only fitness configuration which yields a stable panmictic equilibrium is shown by substituting $F=0, k=0$ into (10) and (11). 
(ii) If $W_{1}=W_{3}$, the stable internal equilibrium is

$$
p=\sqrt{1-2 W_{1}} / 2\left(1-W_{1}\right)
$$

and clearly exists only when $W_{1}<0 \cdot 5$. At this equilibrium $F=2 W_{1}-1$ and is therefore negative.

(iii) Solution (13) required the assumption that $p \neq\left[2\left(1-W_{3}\right)\right]^{-1}$. This follows from substituting $k=0$ into (14) and corresponds to the case when $W_{1}=3 W_{3}-2 W_{3}^{2}$. Contrary to the indication of (13), this fitness structure does lead to a stable equilibrium provided $W_{3}<0 \cdot 5$. The solution is

$$
\begin{gathered}
p=\left[2\left(1-W_{3}\right)\right]^{-1} \\
1-F=\left(1-W_{3}\right)\left[-4 W_{3}\left(1-W_{3}\right)\right. \\
\left.+2\left\{1-2 W_{3}+4 W_{3}^{2}\left(1-W_{3}\right)^{2}\right\}^{1 / 2}\right] /\left(1-2 W_{3}\right) .
\end{gathered}
$$

Apart from these simpler cases, it is cumbersome to solve the cubic analytically. Therefore (15) was solved numerically for a range of $W_{i}$ 's and the stability of each equilibrium thus detected was investigated using the

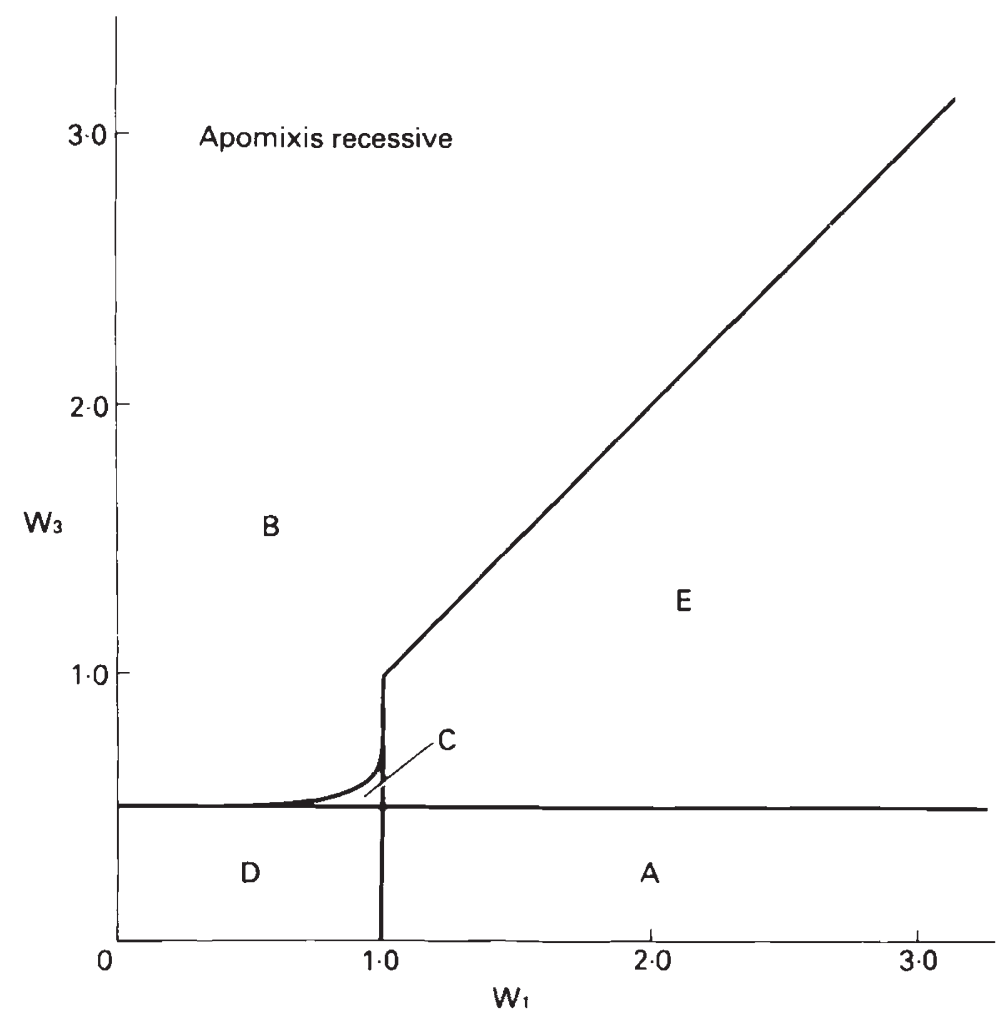

FIG. 1.-Equilibrium phase diagram when the apomictic allele $A_{2}$ is recessive to the random mating allele $A_{1}$. The fitnesses of $A_{1} A_{1}$ and $A_{2} A_{2}$ relative to the heterozygote $A_{1} A_{2}$ are $W_{1}$ and $W_{3}$ respectively. Region $A$ denotes fixation for sexual reproduction, $\mathrm{B}$ fixation for apomictic reproduction, region $C$ polymorphism with fewer than the panmictic proportion of heterozygotes, region $\mathrm{D}$ polymorphism with excess heterozygotes, and region $\mathrm{E}$ fixation for either $\boldsymbol{A}_{1}$ or $\boldsymbol{A}_{2}$. 
recurrence relationshiops (10) and (11). The results are summarized as an equilibrium phase diagram (fig. 1).

Equilibrium phase diagrams were first developed by Hayman (1953) and Hayman and Mather (1953), and used by Workman and Jain (1966) and Asher (1970). They illustrate the types of equilibrium populations for various values of $W_{1}$ and $W_{3}$. The areas marked $\mathrm{A}$ and $\mathrm{B}$ indicate fitness values which lead to populations homozygous for the alternative alleles $A_{1}$ and $A_{2}$ respectively. Thus is area $\mathrm{A}, p=1$ is a stable equilibrium whereas $p=0$ is mutationally unstable, and in area $\mathrm{B}, p=0$ is a stable equilibrium, $p=1$ is unstable. Both areas A and B lack internal equilibria.

The region with at least one stable internal equilibrium is divided for convenience into two areas. Area $C$ is where the equilibrium population would contain fewer heterozygotes than a panmictic population $(F>0)$. Area $\mathrm{D}$ is that fitness set which leads to polymorphic equilibrium heterozygosity in excess of random mating expectations $(F<0)$.

In fig. 1 , region $C$ indicates fitnesses which lead to two internal equilibria one of which is stable, the other being unstable. Thus for $W_{1}=0.9$, $W_{3}=0.55$, the full complement of equilibria is $p=0$, stable; $p=0 \cdot 17647$, $F=0.08858$, unstable; $p=0.71373, F=0.04903$, stable; $p=1$, unstable.

The boundary between regions $\mathrm{B}$ and $\mathrm{D}$ is defined by the line $W_{3}=0.5$ $\left(W_{1} \leqq 0 \cdot 5\right)$. That between $\mathrm{C}$ and $\mathrm{D}$ by the line $W_{3}=0.5\left(W_{1}>0.5\right)$. Between regions $\mathrm{B}$ and $\mathrm{C}\left(W_{1}>0 \cdot 5\right)$ the curve is defined by a curvilinear function of $W_{1}$ and $W_{3}$ such that the two internal equilibria (solutions of (15)) are identical.

When $W_{1}=1 \cdot 0$, and $W_{3} \leqq \frac{1}{2}$ there are no internal equilibria and $p=1$ is stable. If $W_{3} \geqq \frac{2}{3}$, again there are no internal equilibria, but $p=0$ is stable. When $\frac{1}{2}<W_{3}<\frac{2}{3}$ there is one unstable internal equilibrium and $p=0$ or 1 are both stable equilibria (in analogy with a simple one locus selection model with net heterozygote disadvantage). These are also the characteristics of region $\mathrm{E}$.

Overall, fig. 1 confirms the expectation that a mutation for apomixis will increase in a population unless intense selection $\left(W_{3}<0 \cdot 5\right)$ opposes its progress.

\section{(b) Apomixis codominant $\left(k=\frac{1}{2}\right)$}

In the case when $k=\frac{1}{2}$ the polynomial (15) is still fourth degree, and no analytical solutions were obtained. The equilibrium phase diagram (fig. 2) was obtained numerically. For sets of $\left(W_{1}, W_{3}\right)$ near the line $W_{1}=W_{3}>$ $1 \cdot 25$, which separates the region where an apomictic mutant wins (region B), from the region where sex is to some degree protected (region E), many generations of simulation were required to determine the final fate of an apomictic mutant.

In this model, the recursions (3) were used above to determine the initial advantage of an apomictic mutant. Hence a sufficient condition for a codominantly acting apomictic allele to invade a sexual population is that $W_{1}<1 \cdot 25$. Analogous considerations lead to a sufficient condition for the invasion of a sexual mutant into an apomictic $\left(A_{2} A_{2}\right)$ population as $W_{3}<$ $0 \cdot 75$. In general selection for sexuality (high $W_{1}$, low $W_{3}$ ) must be intense to forestall the evolution of apomixis. 


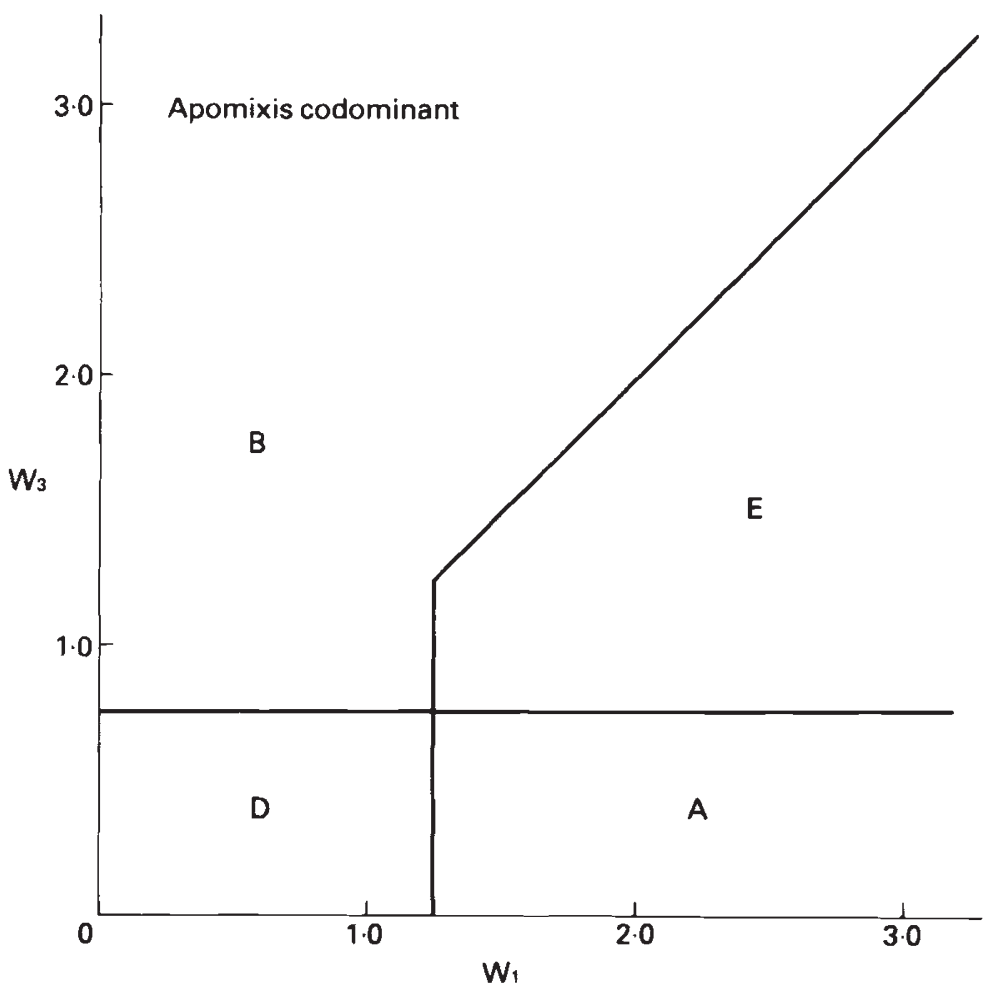

FIG. 2.-Equilibrium phase diagram when the apomictic $\left(A_{2}\right)$ and random mating $\left(A_{1}\right)$ alleles are codominant. Regions $\mathrm{A}, \mathrm{B}, \mathrm{D}$ and $\mathrm{E}$ as for fig. 1 .

(c) Apomixis dominant $(k=1)$

In contrast to the above two cases, when $k=1$ a complete analysis is possible because formula (15) becomes

$$
\left(1-W_{3}\right)(2 p-1)\left(W_{1} p-W_{3}\right)\left[2\left(1-W_{1}\right) p+1\right]=0 .
$$

This equation furnishes four solutions. To be admissible, each solution must be associated with values of $(1-F)$ from equation (13), given $k=1$, such that $0<(1-F)<p^{-1}, q^{-1}$.

(i) If $W_{3}=1$, there is no single equilibrium gene frequency. The population is polymorphic for $A_{1} A_{2}$ and $A_{2} A_{2}$ in neutral equilibrium, and the final gene frequency depends on the original genotype frequencies.

(ii) If $\hat{p}=\frac{1}{2}$ and $F=-1$, the population consists of apomictic heterozygotes. This equilibrium is stable when $\left(W_{1}<1 \cdot 5, W_{3}<1 \cdot 0\right)$, otherwise it is unstable.

(iii) If $p=W_{3} / W_{1}\left(W_{3}<W_{1}\right)$, the restriction on values of $(1-F)$ lead to the following permissible region for this equilibrium.

$$
W_{1} / 2\left(W_{1}-1\right), 1<W_{3}<W_{1} .
$$

This equilibrium is unstable. 


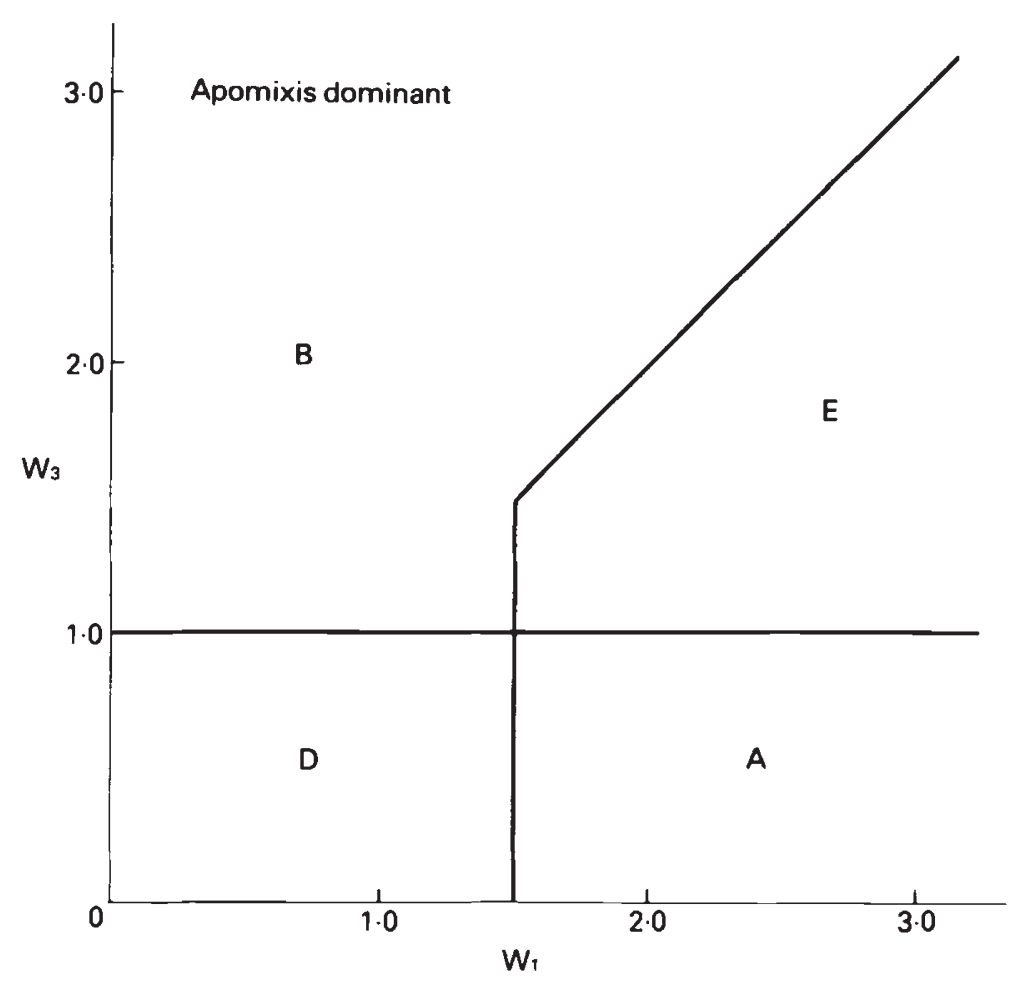

FIG. 3.-Equilibrium phase diagram when the apomictic allele $\left(\boldsymbol{A}_{2}\right)$ is dominant. Regions $A, B, D$ and $E$ as for fig. 1 .

(iv) Solutions of the form $p=1 / 2\left(W_{1}-1\right)$, which because of restrictions on $(1-F)$ yield further unstable equilibria if $1.5<W_{1}<2.0$ since from (13)

$$
(1-F)=2\left(W_{1}-1\right) \text {. }
$$

The genotype $A_{2} A_{2}$ is absent at this equilibrium.

The equilibrium phase diagram in the dominant case is shown in fig. 3 . Four regions are depicted: Region $A$ is where sexuality is fixed and an apomictic population is unprotected against a sexual mutant. Region $\mathrm{B}$ is apomictic (all $A_{2} A_{2}$ ), a sexual population is unprotected against an apomictic mutant. Region $\mathrm{D}$ is also apomictic, and sexuality is unprotected but the population is fixed for $\left(\boldsymbol{A}_{1} \boldsymbol{A}_{2}\right)$. Lastly region $\mathrm{E}$ is the classic "heterozygous" disadvantage region where fixation for either sexuality or apomixis is possible and both kinds of fixed populations are protected against mutation to varying degrees.

\section{Discussion}

The results presented here confirm previous findings (e.g., Maynard Smith, 1971 b; Lloyd, 1977; Charlesworth, 1980) in indicating that apomixis will eventually become fixed in plant populations unless it radically reduces the fitness of its carriers. A similar conclusion was reached by Fisher (1941) 
and subsequent authors (Moran, 1962; Jain and Workman, 1967) in their analysis of the evolution of self-fertilization in plants. These findings raise the question - why are all plants not either inbreeding or apomictic?

The traditional answer to this question for genes governing selfpollination is that inbreeding depression among the selfed progeny provides, except in special circumstances, sufficient loss of fitness to counter the automatic advantage of alleles specifying self-fertilization (Jain, 1976; Maynard Smith, 1977; Frankel and Galun, 1977). However, there are no analogous selective mechanisms to offset the automatic advantage of genes specifying apomixis. This leads to another puzzling question-why are apomictic plants not more common than inbreeding species. Apparently this question has not been seriously discussed in modern evolutionary literature. Yet, it would seem to be of fundamental importance to any real understanding of the evolution of breeding systems in plant. We will therefore use it as the main theme of this discussion.

Clearly, differences in the relative frequency of apomictic and autogamous species among the higher plants must depend on differences in either, or both, their "birth" and "death" rates. The fact that inbreeding species are more common in plants suggests, therefore, that they arise more readily or are lost by extinction much less frequently than apomicts. There is little reason to suppose that agamosperms would be more liable to extinction than inbreeders. Indeed, the opposite may be the case. Newly developed autogamous groups would be more likely to be subject to extinction because of inbreeding depression and exposure in homozygous condition of lethal and deleterious alleles. However, reliable evidence on this point is non-existent.

On the other hand, a strong case can be made that inbreeders and apomicts differ in their "birth" rates. Self-compatible mutants have been found in normally self-sterile species whenever they have been seriously sought. Such appear to occur regularly in plants and often have a relatively simple genetic base (review in Jain, 1976). It seems reasonable to suggest, therefore, that the spread of self-compatibility is checked by the inbreeding depression it induces rather than by the lack of the appropriate mutants. This is certainly the case in Primula (Crosby, 1949; Mather, 1973).

In sharp contrast, apomictic mutants appear to be rare in plant populations. Although they have been eagerly sought in crop species because of their enormous potential for the exploitation of hybrid vigour, few have been found. The only well documented exception is that of Hanna, Schertz and Bashaw (1970) of apospory in a mutant polygynaceous line of grain sorghum. This situation is not entirely unexpected. As early as 1945 , Powers suggested on theoretical grounds that apomixis should be controlled by a number of genes. He argued that a minimum of three genes would be involved in the control of apomixis: One controlling failure of reduction in chromosome number or death of the sexual egg-cell; one controlling development of the unreduced sexual or aposporous egg-cell into an embryo without fertilization, and one controlling presence or absence of fertilization.

The essence of Powers' (1945) suggestion is supported by the available experimental data which indicate that apomixis is often under complex genetic control. All known cases involve at least two loci (Grant, 1971 and Asker, 1979). Simultaneous mutations at two or more loci could be 
expected to be rare in practice. Individual mutants are unlikely to accumulate in populations since each would be at a selective disadvantage without the other complementary member genes controlling the asexual process. On this basis, a lack of suitable mutants would appear to be the major factor hampering the establishment of apomixis in plant populations. The differences in "birth" rates between inbreeders and apomicts would be further reinforced by the fact that apomixis generally has a smaller advantage in inbreeding species (Charlesworth, 1980), so that once a species has become partially or completely self-compatible it is less likely to become apomictic.

The close association between apomixis, polyploidy and interspecific hybridization observed in practice (Stebbins, 1950; Grant, 1971), can be readily explained under this hypothesis. Any apomictic cycle must invoive at least two genetic changes from the normal sexual cycle-one which provides a substitute for meiosis and one which provides a substitute for fertilization. Under the present hypothesis, the prevalence of apomixis among interspecific hybrids would be a consequence of the fact that such hybrids are often partially or completely sterile and this removes the need for one of the simultaneous mutants required for the development of apomixis. Thus, for example, female sterility, could open the way for a single mutation permitting adventitious embryony or somatic apospory and this would lead to the immediate establishment of apomixis in the sterile hybrid.

If this explanation of the greater incidence of apomixis among interspecific hybrid derivatives is valid then it would have important practical implications. In particular, it would indicate that mutagen treated populations of plants which are male fertile but female sterile would be ideal starting material to search for apomictic mutants in crop plants. Female sterile mutants tend to be rare in most collections of genetic stocks, because they are difficult to maintain. Hence, this approach is not without its problems.

However, it would be possible to test the validity of this proposal by exploiting a system such as the two dominant complementary genes for female sterility in grain sorghum described by Casady, Heyne and Weibel (1960). Neither gene has any observable phenotypic effect alone so it should be possible to generate large populations of female sterile plants which, after mutagen treatment, can be screened for fertile mutants. Such mutants could be further screened for evidence of apomictic reproduction using established procedures (Marshall and Brown, 1974; Marshall and Downes, 1977).

Acknowledgements.-We would like to acknowledge the help and encouragement of Drs B. S. Weir, R. N. Oram, and A. J. Pryor, and the reviewer for helpful criticism.

\section{REFERENCES}

ASHER, J. H. 1970. Parthenogenesis and genetic variability. II. One locus models for various diploid populations. Genetics, 66, 369-391.

ASKER, S. 1979. Progress in apomixis research. Hereditas, 91, 231-240.

Batt Agalia, E. 1963. Apomixis. In Recent Advances in the Embryology of Angiosperms, ed. P. Maheshwari, $467 \mathrm{pp}$. International Society of Plant Morphologists, University of Delhi. 
CASADY, A. J., HEYNE, E. G., AND WEIBEL, D. E. 1960. Inheritance of female sterility in sorghum. J. Hered., 51, 35-38.

CHARLESWORTH, B. 1980. The cost of sex in relation to mating system. J. Theoret. Biol. $84,655-671$.

Clausen, J. 1954. Partial apomixis as an equilibrium system in evolution. Caryologia Suppl., $6,469-479$.

CROSBY, J. L. 1949. Selection of an unfavourable gene complex. Evolution, 3, 212-230.

CROW, J. F., AND KIMURA, M. 1965. Evolution in sexual and asexual populations. Amer. Nat., 99, 439-450.

CROW, J. F., AND KIMURA, M., 1969. Evolution in sexual and asexual populations: A reply. Amer. Nat., 103, 89-91.

DARLINGTON, C. D. 1939. The Evolution of Genetic Systems. Cambridge University Press, Cambridge.

DE WET, J. M., AND STALKER, H. T. 1974. Gametophytic apomixis and evolution in plants. Taxon, 23, 689-697.

FISHER, R. A. 1930. The Genetical Theory of National Selection. Clarendon Press, Oxford.

FISHER, R. A. 1941. Average excess and average effect of a gene substitution. Ann Eugen., $11,53-63$.

FRANkel, R., AND GAlun, E. 1977. Pollination mechanisms, Reproduction and Plant Breeding. Springer-Verlag, New York.

GRANT, V. 1971. Plant Speciation, 435 pp. Columbia University Press, New York.

GUSTAFSSON, A. 1946. Apomixis in the higher plants. I. The mechanism of apomixis. Lunds Univ. Arsskr., 43, 1-66.

GUSTAFSSON, A. 1947a. Apomixis in higher plants. II. The causal aspect of apomixis. Lunds Univ. Arsskr., 43, 71-178.

GUSTAFFSON, A., 1947b. Apomixis in higher plants. III. Biotype and species formation. Lunds. Univ. Arsskr., 44, 183-370.

HANNA, W. W., SCHERTZ, K. F., AND BASHAW, E. C. 1970. Apospory in Sorghum bicolor (L.) Moench. Science, 170, 338-339.

HAYMAN, B. I. 1953. Mixed selfing and random mating when homozygotes are at a disadvantage. Heredity, 7, 185-192.

HAYMAN, B. I., AND MATHER, K. 1953. The progress of inbreeding when homozygotes are at a disadvantage. Heredity, 7, 165-183.

HESLOP-HARRISON, J. 1961. A pomixis, environment and adaptation. In Recent Advances in Botany, Vol. I, pp. 891-895. Proc. 9th. Int. Bot. Cong., University of Toronto Press.

JAIN, S. K. 1976. The evolution of inbreeding in plants. Ann. Rev. Ecol. Syst., 7, 469-495.

JAIN, S. K., AND WORKMAN, P. L. 1967. Generalized F statistics and the theory of inbreeding and selection. Nature, 214, 674-678.

KNOX, R. B. 1967. Apomixis. Seasonal and population differences in a grass. Science, 157, 325-326.

KNOX, R. B., AND HESLOP-HARRISON, J. 1963. Experimental control of aposporous apomixis in a grass of the Andropogoneae. Botaniska Natiser, 116, 127-141.

LLOYD, D. G. 1977. Genetic and phenotypic models of natural selection. J. Theor. Biol., $69,543-560$.

MATHER, K. 1973. Genetical Structure of Populations, $197 \mathrm{pp}$. Chapman and Hall, London.

MARSHALL, D. R., AND BROWN, A. H. D. 1974. Estimation of the level of apomixis in plant populations. Heredity, 32, 321-333.

MARSHALL, D. R., AND DOWNES, R. W. 1977. A test for obligate apomixis in grain sorghum R473. Euphytica, 26, 661-664.

MARSHALL, D. R., AND WEIR, B. S. 1979. Maintenance of genetic variation in apomictic plant populations. I. Single locus models. Heredity, 42, 159-172.

MAYNARD SMITH, J. 1968. Evolution in sexual and asexual populations. Amer. Nat., 102, 469-473.

MAYNARD SMITH, J. 1971a. What use is sex? J. Theoret. Biol., 30, 319-335.

MAYNARD SMITH, J. $1971 \mathrm{~b}$. The origin and maintenance of sex. In Group Selection, ed. G. C. Williams Aldine-Atherton, pp. 163-175. Chicago.

MAYNARD SMITH, J. 1976. A short-term advantage for sex and recombination through sib-competition. J. Theor. Biol., 63, 245-258.

MAYNARD SMITH, J. 1977. The sex habit in plants and animals. Lecture Notes in Biomathematics, 19, 315-331, eds. F. B. Christiansen and T. M. Fenchel. Springer-Verlag, New York.

MA YNARD SMITH, J. 1978. The Evolution of Sex, 222 pp. Alden Press, Oxford. 
MORAn, P. A. P. 1962. The Statistical Processes of Evolutionary Theory. Clarendon Press, Oxford.

MULLer, H. J. 1932. Some genetic aspects of sex. Amer. Nat., 8, 118-138.

NYGREN, A. 1954. Apomixis in angiosperms. II. Bot. Rev., 20, 577-649.

NYGRen, A. 1967. Apomixis in the angiosperms. Handb. der Pflanzenphys., 18, 551-596.

POWERS, L. 1945. Fertilization without reduction in guayule (Parthenium argentatum Gray)

and a hypothesis as to the evolution of apomixis and polyploidy. Genetics, 30, 323-346.

SARAN, S., AND DE WET, J. M. J. 1970. The mode of reproduction in Dicanthium intermedium (Gramineae). Bull. Torry Bot. Club, 97, 6-13.

STEBbiNS, G. L. 1941. Apomixis in the angiosperms. Bot Rev., 10, 507-542.

STEBBins, G. L. 1950. Variation and Evolution in Plants, 643 pp. Columbia University Press, New York.

WILliamS, G. C. 1975. Sex and Evolution. Princeton University Press, Princeton N.J.

WILliams, G. C., AND MitTon, J. B. 1973. Why reproduce sexually? J. Theoret. Biol., 39, 545-554.

WORKMAN, P. L., AND JAIN, S. K. 1966. Zygotic selection under mixed random mating and self-fertilisation: Theory and problems of estimation. Genetics, 54, 159-171. 